\title{
MULTI EXPOSURE IMAGE FUSION USING SYNCHROSQUEEZING TRANSFORM
}

\author{
Libya Thomas and Anoop K Johnson \\ Mar Baselios College of Engineering and Technology, Trivandrum, India.
}

\begin{abstract}
A novel method for fusion of images with multiple exposure is proposed in this paper. Synchrosqueezing transform which gives more concentrated representation of a given signal. which is used for analyzing $1 D$ signals are extended to $2 D$ signals like images and further they are used for applications like multi-exposure image fusion where various images of a particular scene with different exposure is fused to produce an image with better exposure than all the input images given. Curvelet based synchrosqueezing transforms are used when the domain is changed from $1 D$ to $2 D$. The numerical results show the proposed method produced an image which has better exposure and quality than all the input images given.
\end{abstract}

Keywords: Synchrosqueezing Transform, curvelet transform, synchrosqueezing energy, energy cluster.

Cite this Article: Libya Thomas and Anoop K Johnson, Multi Exposure Image Fusion Using Synchrosqueezing Transform, International Journal of Advanced Research in Engineering and Technology, 10 (3), 2019, pp 169-183.

http://iaeme.com/Home/issue/IJARET?Volume $=10 \&$ Issue $=3$

\section{INTRODUCTION}

It is very familiar that a signal can be modeled or analyzed by using various transforms. A transform is a method which converts a raw signal to a processed signal. Various commonly used transforms include Fourier transform, Short Time Fourier transform (STFT), Continuous wavelet transform and so on. While dealing with Fourier transform one know that it converts a signal to its frequency components or it decomposes a signal to various frequency components that makes up that particular signal. Even though it is widely used it has got a drawback ie. It is applicable only for stationary signals. And thus, for signals like non stationary signals we can employ STFT -short time fourier transform.

STFT is a window-based approach which analyze a given signal by splitting it into small regions, so that within that small region the non-stationary signal appears to be a stationary signal. But it has also hot a disadvantage that is it does not give time localization as well as frequency localization simultaneously. If one requires more precision on time some compromise has to be made in frequency and if frequency resolution is required the time scale has to be compromised. And this is with respect to the Heisenberg's uncertainty principle.

In order to overcome with this issue wavelet transforms were introduced. 
In a wavelet transform we use mother wavelets to analyze a signal. The mother wavelet can be changed with respect to scale as well as position by changing the scaling and position parameters of the mother wavelet. A mother wavelet can be represented as

$$
\varphi_{a, b}(t)=\frac{1}{\sqrt{a}} \varphi\left(\frac{t-a}{b}\right)
$$

All the above methods have its own advantages as well as drawbacks but still all the methods gives a time-frequency or time-scale representation of a given signal. By increasing the concentration of time-frequency representation gives a sharpened plot which gives a welldefined explanation for our signal that has to be analyzed. And one such methods which are used to sharpen the time frequency representation include synchrosqueezing transform. (SST) $[1][2]$.

It is a kind of reassignment method [3] which finally resulted in synchrosqueezing transform. Synchrosqueezing transform is thus a special kind of reassignment method which reallocates the coefficients which resulted from any operations like fourier transform, STFT and so on to a new scale. Mainly from time-scale to time-frequency. The entire energy along the signal willget concentrated on a single point or around a line across the signal. Since all energy is getting concentrated to certain points, hence the name synchrosqueezing.

Synchrosqueezing can be performed by using either continuous wavelet transforms as well as short time fourier transform. Transform based on continuous wavelet transform was first developed and then the concept was extended to STFT. There is a good trade-off between choosing STFT and continuous wavelet transform (CWT) to perform synchrosqueezing transform. STFT based synchrosqueezing transform can handle a large or wide range of modulations with low frequencies and CWT based SST acts satisfactorily for higher frequencies. One of the main applications of SST is in the decomposition of multicomponent signals. This particular SST is further extended toan application in image processing i.e. To fuse different images of a single scene which has different exposure and produce a single image in which the exposure is even and all objects can be fully visible. The pattern of the paper is as follows: section 2 briefs on the application of STFT on image,section 3 deals with STFT based SST ,section 4 describes the application of SST for image fusion and finally section 5 gives the numerical results.

\section{APPLICATION OF STFT ON AN IMAGE}

The short time fourier transform is said to be a linear time frequency method which can identify the changes that occurred in a signal with respect to time. When comparing with fourier transform, fourier transform does not provide the time information of the frequency components present in a given signal. But the frequency information can be obtained when the signal is prewindowed and analyzed. In short during performing STFT the signal is divided into small sections using window functions and FFT is computed on each section. A Gaussian function can be a good choice for window. The STFT of a ID signal can be represented as:

$$
X(t)=\int_{-\infty}^{\infty} x(t) w(t-\tau) e^{-j w t} d t
$$

Similarly on applying STFT on a $2 \mathrm{D}$ signal i.e. let it be an image $f(x, y)$. Then can be computed as:

$$
X\left(\tau_{1}, \tau_{2}\right)=\iint_{-\infty}^{\infty} f(x, y) w\left(x-\tau_{1}, y-\tau_{2}\right) e^{-j w x} e^{-j w y} d x d y
$$

Here $\mathrm{w}(\mathrm{t})$ is the window used. A Gaussian window with a width $\sigma$ can be represented as:

$$
G_{\sigma}(x, y)=\frac{1}{\sqrt{2 \pi \sigma^{2}}} \exp \left(-\frac{x^{2}+y^{2}}{2 \sigma^{2}}\right)
$$


A $15 \times 15$ window size can be opted. Always it is convenient to choose a window with smaller size since image will be stationary within that window. As the window size is larger signal will no longer be stationary.

\section{STFT BASED SYNCHROSQUEEZING TRANSFORM}

Synchrosqueezing transform was introduced as a special type of reassignment method. The coefficients resulting from various transforms are reallocated to a different point by taking into account the instantaneous frequency of each mode. Instantaneous frequency is similar to that of a fourier frequency. It represents the highest oscillating component or frequency in each mode.

Usually CWT was the transform or the preprocess that was done inorder to perform synchrosqueezing. The short time fourier transform of a signal is computed. The coefficients obtained by using STFT is reassigned to a new point in time-frequency plane, which represents the instantaneous frequency of that particular signal. The instantaneous frequency from the coefficients can also be obtained by taking the derivative of the coefficients or by the equation that is used to find IF is as follows:

$$
w_{f}(a, t)=\frac{1}{2 \pi} \frac{\partial}{\partial t} \arg X(a, t)
$$

i.e. the coefficients of $\mathrm{X}(\mathrm{t})$ is moved from $(\mathrm{a}, \mathrm{t})$ to $\left(w_{f}(a, t), t\right)$. The equation 5 represents the instantaneous frequency at time $t$ and it is filtered at frequency $\varphi$. Once the coefficients are reallocated the SST is calculated at every interval of $w_{f}(\varphi, t)=\omega$ and it can be represented using the formula:

$$
S_{f}(\omega, t)=\frac{1}{g(0)} \int_{R} X(a, t) \delta\left(\omega-w_{f}(\varphi, t)\right) d a
$$

Here $\mathrm{g}$ is the window function which is used.

\section{APPLICATION OF SST FOR IMAGE FUSION}

So far SST was only applied to one dimensional multi-component signals where each mode present on the signal is extracted and analyzed. When moving to images we are extending the application of SST to a multidimensional domain, where each and every characteristics of a multi-dimensional data is analyzed. There may be different modes present for a single pair of points say $(\mathrm{x}, \mathrm{y})$. When dealing with 2D signals a simple approach was to combine 2D wavelet transform with synchrosqueezing transform and then find out the wave vector at each location. But for an image the components present in it can have different wave vectors at each point which is similar to a 1D signal. SST cannot distinguish the different components which share the same wave number and having different wave vectors. The reason behind this drawback is the isotropic property of the wavelet transform.

To overcome this issue synchrosqueezedcurvelet transform (SSCT) shall be employed.

\subsection{Synchrosqueezedcurvelet transform}

A curvelet transform mainly depends on two parameters, the radial scaling parameter $t$ as well as the angular scaling parameter $s$. it is given that both the parameters should satisfy $1 / 2<s<$ $t<1$.[4], so that an accurate estimate of wave vector is obtained for a given wave number. When $s=t$ then we can say the curvelet transform generally becomes a wave packet [5]. If we consider that we have a signal then the synchrosqueezedcurvelet transform can be performed by using the following steps: 
1. Thecurvelet function or transform of a given signal is computed say let it be represented as $w_{f}(a, \theta, b)$

$$
w_{f}(a, \theta, b)=\left\langle w_{a \theta b}, f\right\rangle=\int_{R^{2}} \overline{w_{a \theta b}(x)} f(x) d x
$$

Where $\mathrm{a} \in[1, \propto), \theta \in[0,2 \pi), b \in R^{2}$ and

$$
w_{a \theta b}=\int a^{\frac{t+s}{2} e^{2 \pi i a(x-b) e \theta} \omega\left(A_{\theta^{R}}{ }^{-1}(x-b)\right)}
$$

Here $A_{\theta}$ is the scaling matrix frowm which the value of a, which is the distance between the center of the curvelet and fourier domain is obtained.

$$
A_{\theta}=\left(\begin{array}{cc}
a^{t} & 0 \\
0 & a^{s}
\end{array}\right)
$$

And $R_{\theta}$ is the rotation matrix.

$$
R_{\theta}=\left(\begin{array}{ll}
\cos \theta & -\sin \theta \\
\sin \theta & -\cos \theta
\end{array}\right)
$$

2.The support of $w_{f}(a, \theta, b)$ is then computed which separates each of the components present in the signal.

3.For each $\mathrm{b}$ we can find an estimate of the local wave vector and is defined as

$$
v_{f}(a, \theta, b)=\frac{\nabla_{b} w_{f}(a, \theta, b)}{2 \pi i w_{f}(a, \theta, b)}
$$

The wave vectors are estimated independent to the position $b$ and if the coefficients with same $v_{f}$ are reallocated to the same point then it gives a concentrated or sharpened representation of the signal.

4. Thesynchrosqueezing energy is concentrated around $v_{f}(a, \theta, b)$ and thus the energy can be computed as:

$$
T_{f}(v, b)=\int\left|w_{f}(a, \theta, b)\right|^{2} \delta\left(R v_{f}(a, \theta, b)\right) a d a d \theta
$$

5. Finally each modes present are decomposed.

\subsubsection{Curvelet on an Image}

When comparing fourier transform wavelet transform and curvelettansform, fourier transform it gives the time information of a signal in time. Wavelet transform gives well localized representation of a signal both in time as well as frequency. Fourier transform gives localization only in frequency.

Curvelet transform is a higher dimensional generalization of wavelet transform. And it represents an image in various scales as well as angles. It handles discontinuities present in an image with perfection. The general curvelet transform is as represented in the figure 1. One of the main applications of curvelet transform is denoising of image.

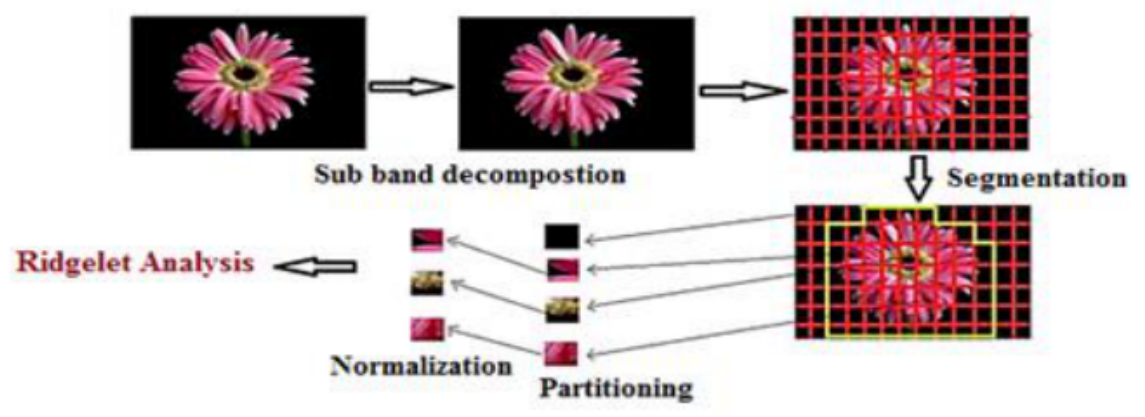

Figure 1 Curvelet Transform 
The image is first segmented by using sub band decomposition which is then followed by partitioning which partitions those areas of the image which contains objects and those portions are further analysed using the ridglet analysis. Ridglet analysis is done by using the Radon transform.

\subsubsection{SynchrosqueezedCurvelettransform On Image}

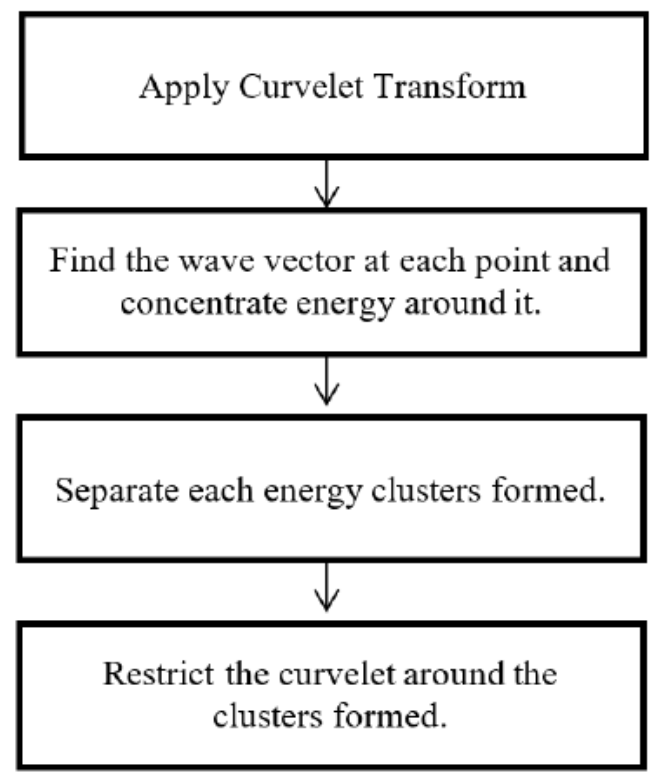

Figure 2 Flow chart of SSCT on image

The curvelet transform is applied on to the image and the coefficients obtained by using the synchrosqueezedcurvelet transform is then remapped into the energy clusters which were obtained. And it gives a concentrated representation of the important portions of the image. The remaining portions remains ingnored and those portions with better quality and information will get even highlighted.

Once the forward transform is done then the inverse is obtained or the original image is reconstructed by performing the inverse operations. The flow chart of the entire process is given below in fig 2

\subsubsection{Multi Exposure Fusion}

Input images

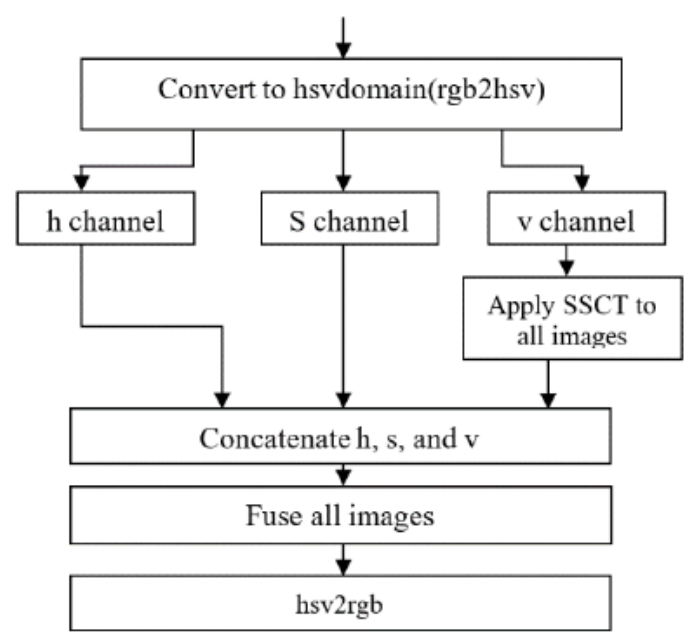

Figure 3. Fusion using SSCT in hsv domain 
Once the synchrosqueezing transform is obtained the resulting image will have certain portions highlighted and other portions remain unprocessed. We have different images of an particular scene which has different exposure. Each image after processed will have different areas highlighted. A particular area which is been processed in one image may be ignored in other and the portion ignored in the first may be the portion considered in the second image.

Thus all the images are further fused by considering only those portions which are considered and processed.

In order to apply the synchrosqueezingcurvelet transform on image the image should be of $2 \mathrm{D}$ in size.

Input images

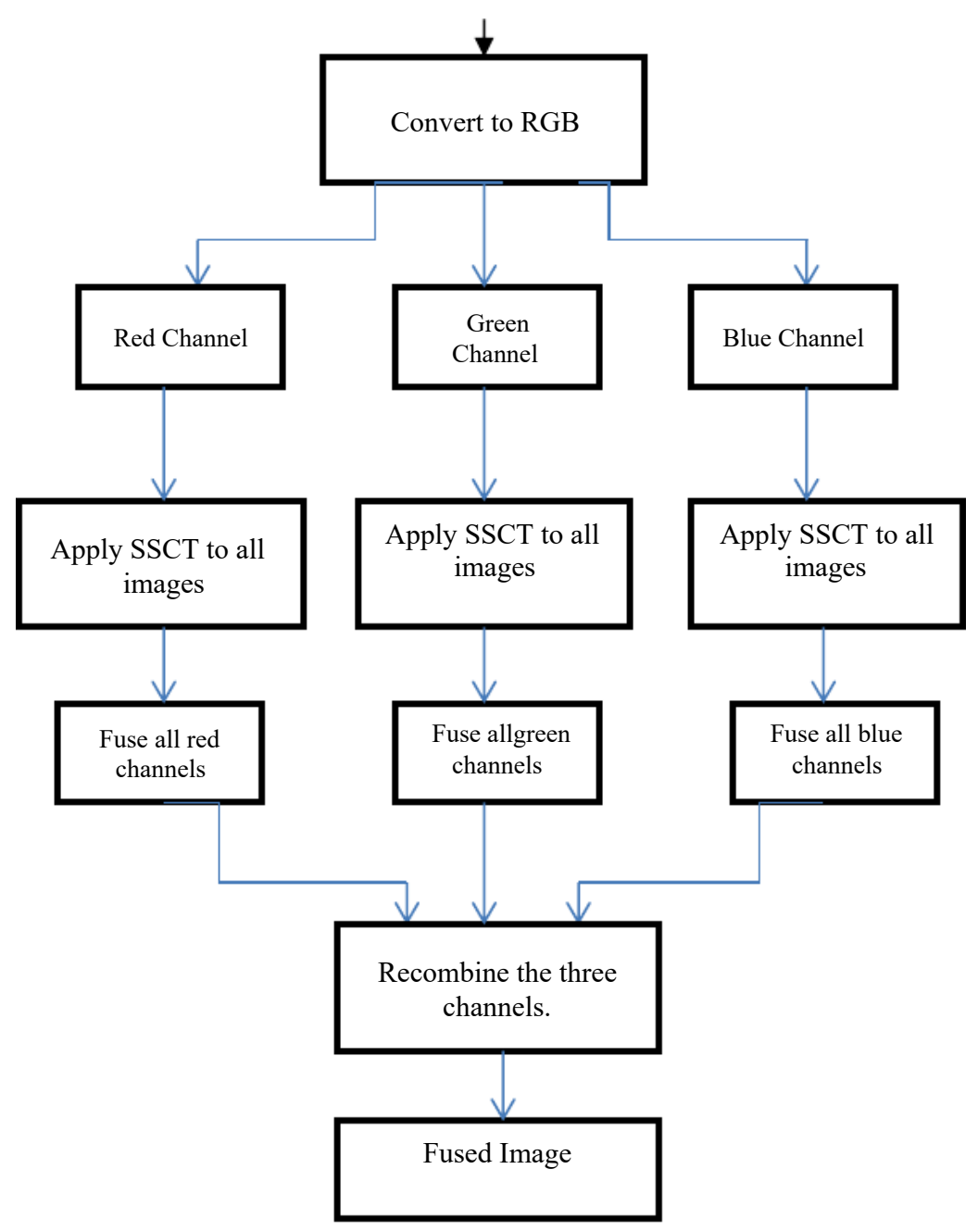

Figure 4 Image Fusion using SSCT in rgb domain

So in order to perform the transform on a colour image the image is first divided into different colour channels like red, green and blue and perform the operations separately an each channels separately perform fusion on each channel and finally combine the three channels together to get the fused image. All the images with different exposure is given as input to the block diagram. Also at the same time image can also be converted to hsv domain and perform the same operations on $\mathrm{v}$ and then finally combine the $\mathrm{h}, \mathrm{s}$ and $\mathrm{v}$ channels together. Which are represented in fig 3 and fig 4.

And the final output obtained will be the one which contained all the processed portions form all the images which produced a good quality which better exposure than all the input images. The fusion of the processed images can be performed based on wavelet fusion. 


\section{NUMERICAL RESULTS}

The proposed method was applied on a three sets of four images which has different exposure were the first image in every set is least exposed that is it is the darkest image and the brightness increases along the second and third images and finally fourth image is over exposed image were most of the portions on the image appears to be white. The simulations were done using the software MATLAB and results were obtained.

The fused output images were obtained which contains neither darker portion as in every first image nor brighter portions as in image four. A perfect blend of four images were obtained in order to obtain the fused images.

The various parameters that are used for the measure of quality of the output image obtained are:

1. Entropy: The randomness of a given image is measured using entropy values. It is a useful measure in the terms of image coding. Entropy values shall be obtained with the help of histograms of the image as the histogram it gives probabilities of different grey levels. Entropy is also used for automatic focusing of a given image.

Entropy was first defined by Shannon. Basically entropy is assumed as a measure which gives idea about the amount of information content present in the given image. Sometimes the content of information present in the image which is nothing but the entropy is interpreted as the uncertainty about the source of the information content.

The state of intensity levels that a particular pixel in an image can handle or adopt can also be a definition for entropy. The entropy value can provide a better image comparison details and hence it can be used as a good performance analysis measure.

2. PSNR: It computes the peak signal to noise ratio of an image say original image with a reference image. As the PSNR value is higher better will be the image. The first step to find the PSNR value is to compute the mean square error which has to be lower for better image quality.

The PSNR value is calculated by using the maximum value of the pixels that is present in a given image. If the given image is a eight bit image then the maximum pixel value is 225 .

When applied to a color image the PSNR is calculated in the same way as of a gray scale image. But the only difference is that the MSE value is calculated by taking the difference between values in each if the color channels that is red channel, blue channel and green channel. That is the image is converted to different color spaces and then PSNR is obtained.

3. MSE: Mean Square Error compares the difference between two images say one the reference image and the other original image directly by computing the variations in the pixel values. For an image which has good quality the MSE value must be lower and almost close to zero. The spectral quality if the fused image is indicated by using the MSE value.

Image compression quality can be analyzed by using MSE value. It computes the two error metrics one that of the compressed image and the other the original image which we have. In other words it can be defined as the sum odf the squared error between the two images which is used for comparison.

Usually the value of PSNR and MSE value are analyzed. For an image which is reconstructed form some other input images the the PSNR value has to be higher for the reconstructed image and the MSE value must be lower for the image to have better quality. So we need to analyze whether the image reconstructed have lower MS value and higher PSNR value.

4. SSIM: Structural similarity index measure it compares two images and gives the difference between the two images. It is one of the full reference method. As defined it compares two images one the reference image which is assumed to have perfect quality and other the original 
image or here let it be the fused image. It is one of the image quality assessment method. It was introduced to improve the other traditional methods like PSNR, MSE etc. SSIM is a perception based method. It compares the structural difference occurred in an image with respect to a reference image. Structural information in other words shall be defines as the strong inter dependencies between the pixels present in the image.

5. CC: Correlation Coefficient is a measure which gives the relationship that exist between two quantities. When considering the image each and every pixel present in the given two images are compared and the correlation between them are obtained. So that this measure can be used to find the measure of difference between the two images compared. The highest value it can take is 1 and the lowest value is 0 . The correlation coefficient having a value 1 represents that the images compared are entirely different and the lowest value shows that the images compared are similar.

Certain benefits of using Correlation coefficient include, it finally gives a single scalar value which represents the difference or comparison between two images and the scalar value say $r$ is not sensitive to variations in brightness as well as contrast occurring across the images.

Correlation Coefficient (CC) and SSIM can be defined as image recognition methods.

5. SSIM and Correlation Coefficient values even though different variables somehow, they share the same meaning and either one can be employed. Hence SSIM value is used here.

The quality of the output was verified based on visual analysis as well as by obtaining the entropy of each of the input images as well as that of the output image. PSNR value MSE, CC and the similarity index value (SSIM) of each of the input and output images was also obtained. All the results obtained are illustrated on the table $1,2,3,4,5,6$ and 7 and fig $5 \mathrm{c}, 6 \mathrm{c}$ and $7 \mathrm{c}$ given below.

Entropy represents the randomness of the pixels and it represents the amount of information present in the given signal the case here is the image.

SSIM represents the similarity between two images. Its gives on measure of an image degraded from other. Unlike other measures ssim gives a visual structure of an image. The highest ssim score that can be obtained is 1 and a score of 1 denotes the images are similar. The ssim values are displayed in table 7.

Each of the input images are compared with the output image and the value obtained shows that the input and output images are entirely different.

By analyzing the table it can be concluded that the obtained output image has got a better quality on the grounds of entropy as well as psnr that the input images and also has obtained better exposure than the input images. SSIM values as wll as the correlation coefficient values shows that a new image is produced from the input images given.

The figure $5 . \mathrm{a}, \mathrm{b}$ figure $6 \mathrm{a}, \mathrm{b}$ and $7 \mathrm{a}, \mathrm{b}$ shows the input and output images obtained.

\section{Image set 1}

Image set one has four set of images of a house taken at different period of time. As can be seen the first image is darker and most of the portions appears black in color. And the brightness or the exposure of the image increases as we move towards the forth image. It can be seen that the forth image is over exposed and most of the portions appears white in color.

All the four are fused using the proposed method and the portions visible in all the images are combined together. That is a portion which is visible may not be visible in other but it has some other portions which is perfectly visible. So to obtain a fused image in which all the portions are perfectly visible.

Input images 

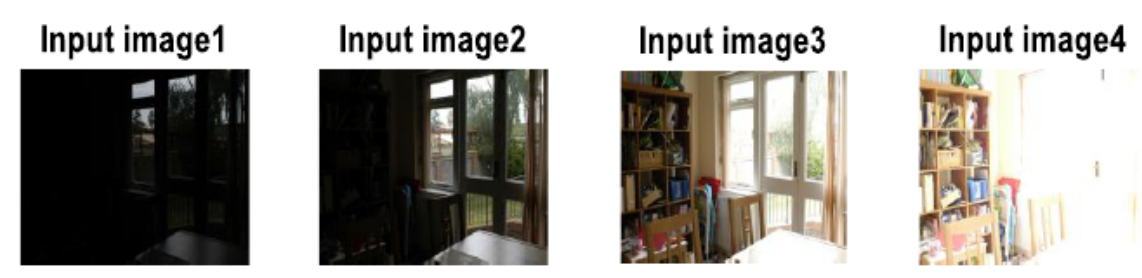

Figure 5 a. Set 1 Input Images

Output obtained

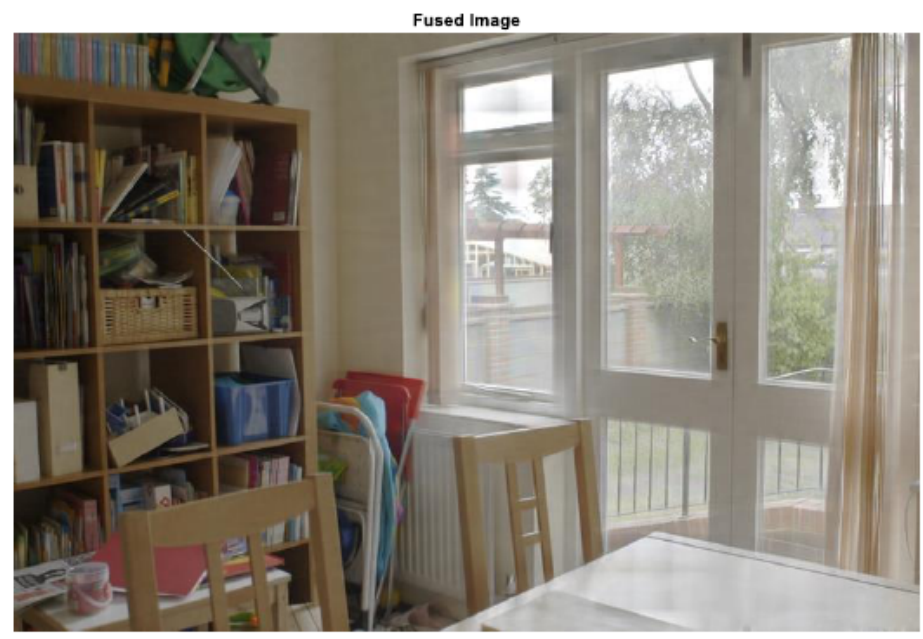

Figure 5 b. Set 1 Fused image

Table 1 gives the entropy values of the input images and the fused image for the first set of images. From the values obtained a bar graph which represents the same is also illustrated in figure $7 \mathrm{c}$. value.

By analyzing the graph the bar which represents the fused image has got the highest entropy

Table 1

\begin{tabular}{|c|c|}
\hline No: & Entropy(bits/pixel) \\
\hline Image1 & 3.7719 \\
\hline Image2 & 6.5720 \\
\hline Image3 & 7.4656 \\
\hline Image4 & 4.4376 \\
\hline Fused image & 7.7101 \\
\hline
\end{tabular}




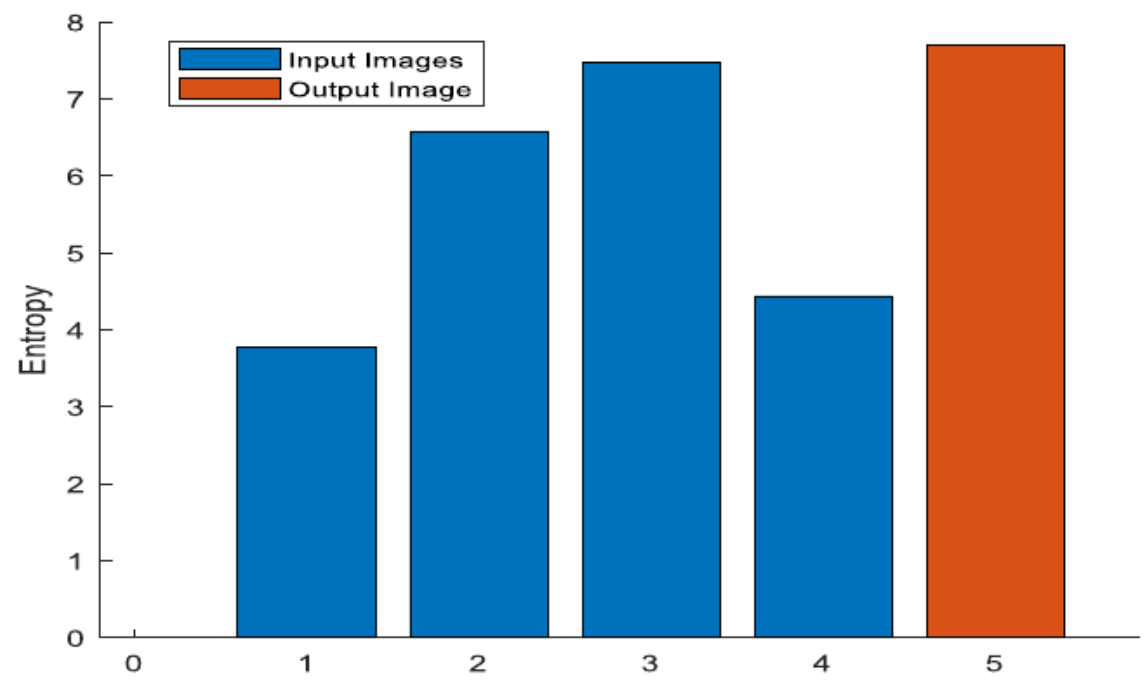

Figure 5 c. Entropy plot of image set 1

Table 2 shows a comparison of the PSNR as well as the MSE values of all the input images and output images of first set of images.

The PSNR value is larger for the fused image at the same time the MSE value is lesser for the fused image compared to other input images.

The graph obtained from the PSNR and MSE values for the first set of image is shown in figure $5 \mathrm{~d}$

Table 2

\begin{tabular}{|c|c|c|}
\hline No: & PSNR(dB) & MSE \\
\hline Image1 & 20.2404 & 0.0095 \\
\hline Image2 & 20.7942 & 0.0082 \\
\hline Image3 & 21.3960 & 0.0072 \\
\hline Image4 & 20.6383 & 0.0087 \\
\hline Fused image & 22.1785 & 0.0061 \\
\hline
\end{tabular}
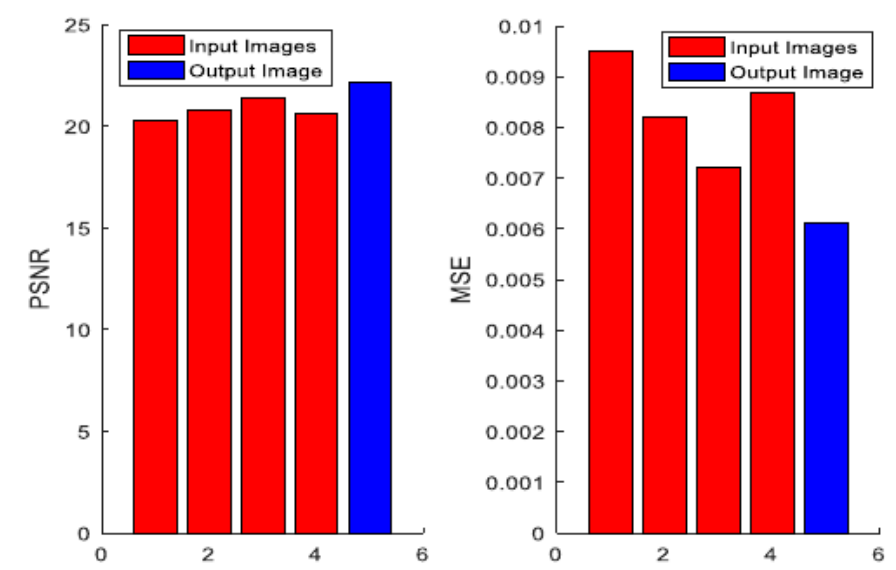

Figure 5 d. PSNR and MSE plot of image set 1 


\section{Image set 2}

As in the case of image set 1 set 2 also has four set of images of a baby taken at different exposure. As can be seen the first image is darker and most of the portions appears black in color.

And the brightness or the exposure of the image increases as we move towards the forth image. It can be seen that the forth image is over exposed and most of the portions appears white in color.All the four are fused using the proposed method and the portions visible in all the images are combined together. That is a portion which is visible may not be visible in other but it has some other portions which is perfectly visible. So to obtain a fused image in which all the portions are perfectly visible.

Input images

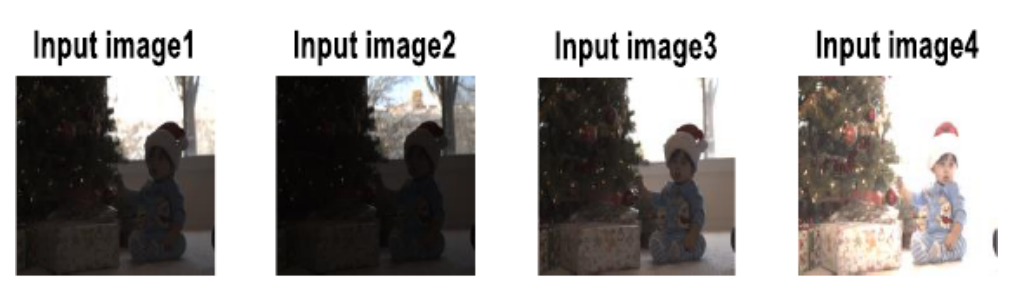

Figure 6 a set 2 input images

Output obtained

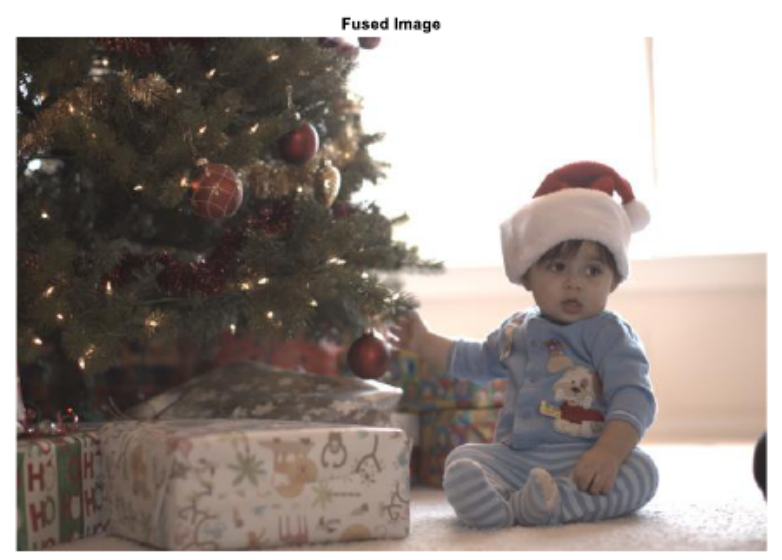

Figure $6 \mathrm{~b}$ Set 2 Fused image

Table 3 gives the entropy values of the input images and the fused image for the second set of images. From the values obtained a bar graph which represents the same is also illustrated in figure $6 \mathrm{c}$. By analyzing the graph the bar which represents the fused image has got the highest entropy value. 
Table 3

\begin{tabular}{|c|c|}
\hline No: & Entropy(bits/pixel) \\
\hline Image1 & 6.3028 \\
\hline Image2 & 6.2014 \\
\hline Image3 & 6.4939 \\
\hline Image4 & 5.6416 \\
\hline Fused image & 7.2625 \\
\hline
\end{tabular}

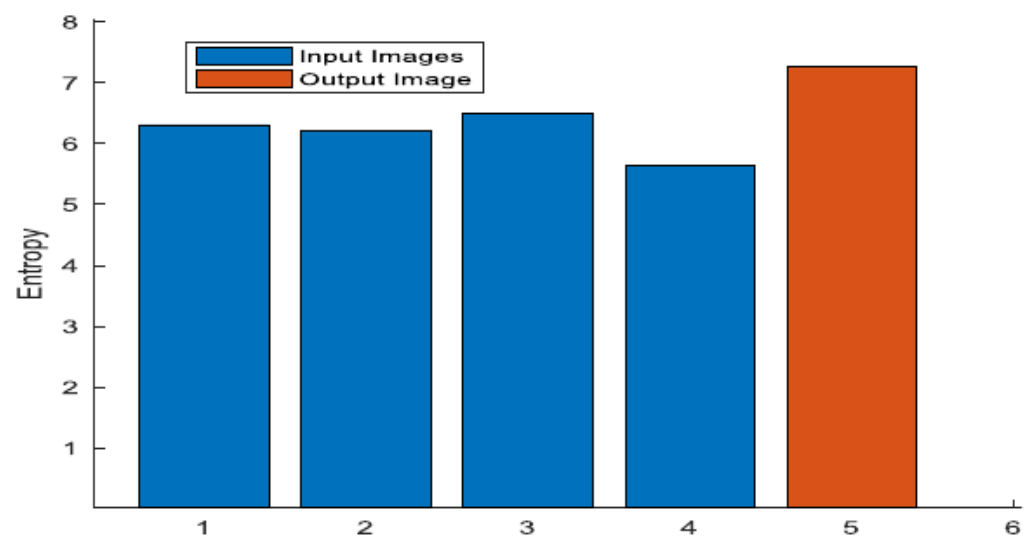

Figure 6 c. Entropy plot of image set 2

Table 4 shows a comparison of the PSNR as well as the MSE values of all the inputimages and output images of second set of images. The PSNR value is larger for the fused image at the same time the MSE value is lesser for the fused image comparedto other input images.

The graph obtained from the PSNR and MSE values for the second set of image is shown in figure $6 \mathrm{~d}$

Table 4

\begin{tabular}{|c|c|c|}
\hline No: & PSNR(dB) & MSE \\
\hline Image1 & 20.9116 & 0.0076 \\
\hline Image2 & 20.7661 & 0.0083 \\
\hline Image3 & 21.3714 & 0.0075 \\
\hline Image4 & 21.2768 & 0.0072 \\
\hline Fused image & 21.5728 & 0.0070 \\
\hline
\end{tabular}
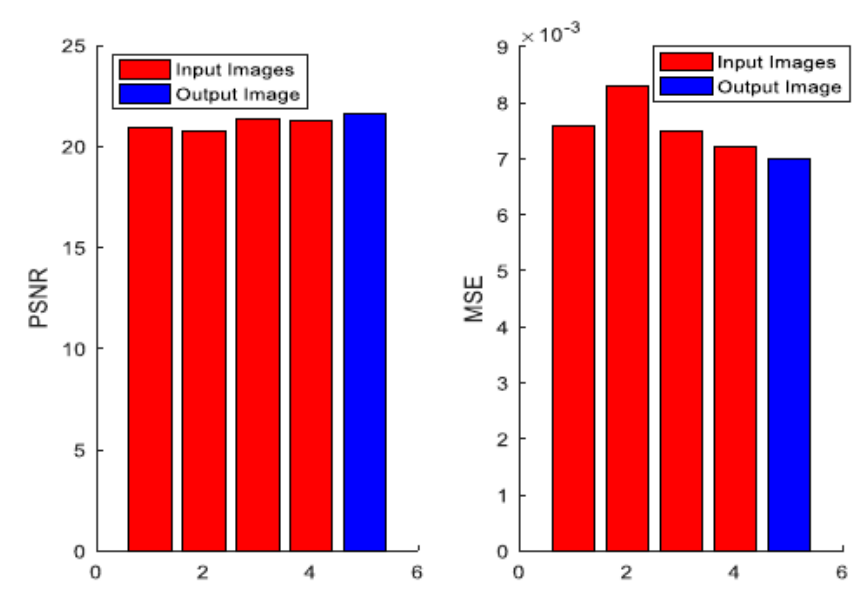

Figure $6 \mathrm{~d}$ PSNR and MSE plot of image set 2 


\section{Image set 3}

Image set three also has four set of images of a man playing piano taken at different exposure. As can be seen the first image is darker and most of the portions appears black in color.

And the brightness or the exposure of the image increases as we move towards the forth image. It can be seen that the forth image is over exposed and most of the portions appears white in color.

All the four are fused using the proposed method and the portions visible in all the images are combined together. That is a portion which is visible may not be visible in other but it has some other portions which is perfectly visible.

So to obtain a fused image in which all the portions are perfectly visible.

Input images
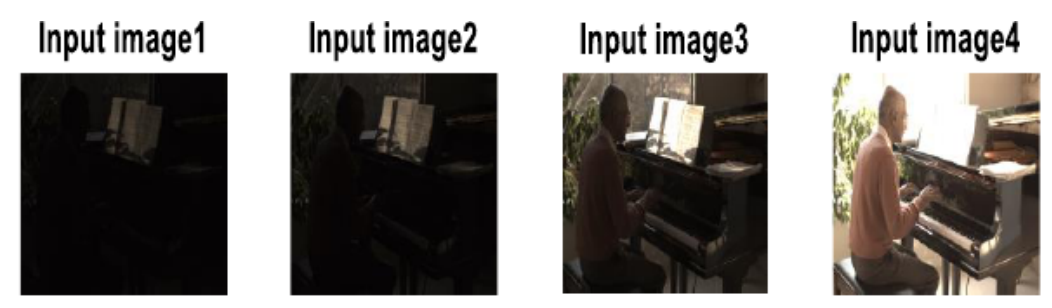

Figure 7 a set 3 input images

Output obtained

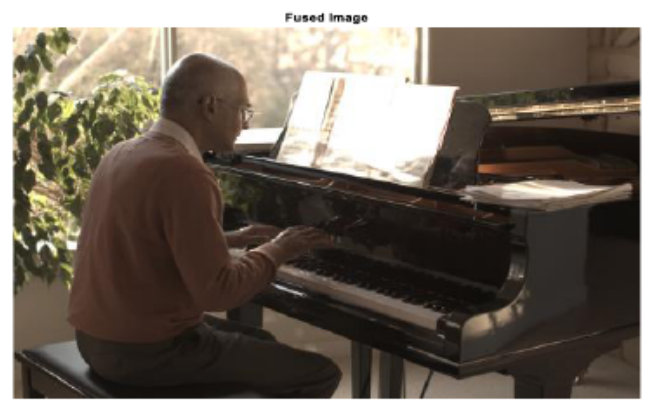

Figure 7 b Set 3 Fused image

Table 5 gives the entropy values of the input images and the fused image for the second set of images. From the values obtained a bar graph which represents the same is also illustrated in figure 7c. value.

By analyzing the graph the bar which represents the fused image has got the highest entropy

Table 5

\begin{tabular}{|c|c|}
\hline Image No: & Entropy (bits/pixel) \\
\hline Image1 & 4.1790 \\
\hline Image2 & 5.0122 \\
\hline Image3 & 6.6183 \\
\hline Image4 & 6.7669 \\
\hline Fused image & 6.9658 \\
\hline
\end{tabular}




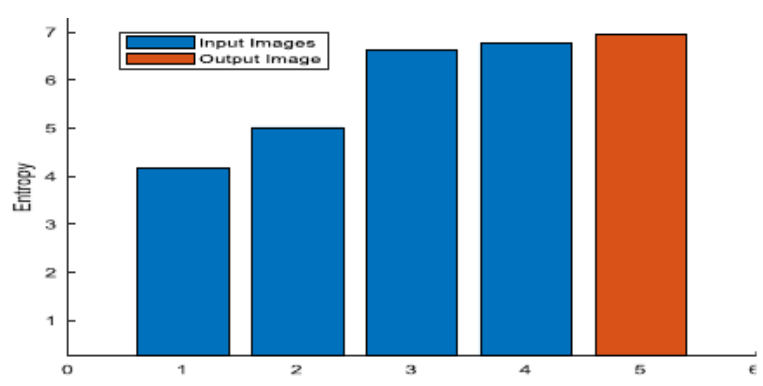

Figure 7 c. Entropy plot of image set 3

Table 6 shows a comparison of the PSNR as well as the MSE values of all the input images and output images of third set of images.

The PSNR value is larger for the fused image at the same time the MSE value is lesser for the fused image compared to other input images. The graph obtained from the PSNR and MSE values for the third set of image is shown in figure $7 \mathrm{~d}$

Table 6

\begin{tabular}{|c|c|c|}
\hline Image No: & PSNR (dB) & MSE \\
\hline Image1 & 20.2814 & 0.0100 \\
\hline Image2 & 20.4375 & 0.0091 \\
\hline Image3 & 21.0932 & 0.0076 \\
\hline Image4 & 21.5627 & 0.0069 \\
\hline Fused image & 21.7649 & 0.0061 \\
\hline
\end{tabular}
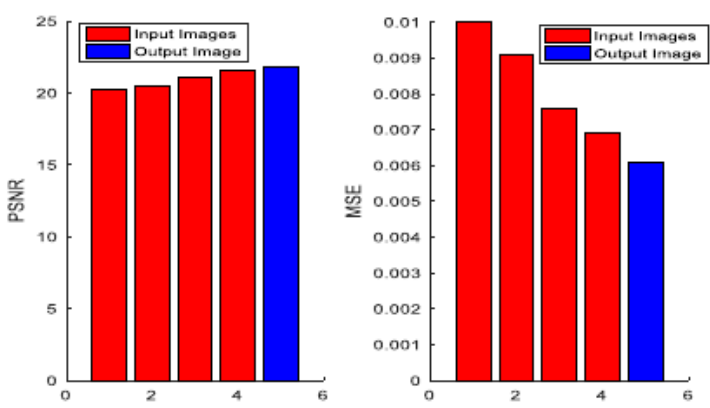

Figure $7 \mathrm{~d}$. PSNR and MSE plot of image set 3

Table 7

\begin{tabular}{|c|c|c|c|}
\hline Image No: & Set 1 & Set 2 & Set 3 \\
\hline Image1 & 0.6907 & 0.0105 & 0.1319 \\
\hline Image2 & 0.1691 & 0.0360 & 0.0859 \\
\hline Image3 & 0.0070 & 0.0026 & 0.0126 \\
\hline Image4 & 0.0036 & 0.0002 & 0.0006 \\
\hline
\end{tabular}

\section{CONCLUSION}

The curveletbased synchrosqueezing transform is applied on to 2D signals that is on images and it is used for the various applications and one such applications is multi-exposure image fusion which is presented in the paper.

The entire experiments were done by converting into hsv domain, then processing and finally converting it back into rgb domain. Various parameters were considered to check the quality of the image. And the parameters analyzed was entropy, psnr and SSIM. The numerical 
results show that the image produced using the proposed method shows quality in terms of entropy as well as psnr than the input images given. It was also analyzed based on visual basis and was analyzed to have better exposure than the input images.

\section{REFERENCES}

[1] I. Daubechies and S. Maes, "A nonlinear squeezing of the continuous wavelet transform based on auditory nerve models," Wavelets in medicine and biology, pp. 527-546, 1996.

[2] I.Daubechies, J .Lu, and H.-T. Wu, "Synchrosqueezed wavelet transforms: an empirical mode decomposition-like tool,"Applied and Computational Harmonic Analysis, vol. 30, no. 2, pp. 243-261, 2011.

[3] K. Kodera, R. Gendrin, and C. Villedary, "Analysis of time-varying signals with small bt values," IEEE Transactions on Acoustics, Speech, and Signal Processing, vol. 26, no. 1, pp. 6476, 1978.

[4] HaizhaoYangy and Lexing Ying, Synchrosqueezedcurvelet transform for 2d mode decomposition. Submitted on 22 Oct 2013, arXiv:1310.6079

[5] H. Yang and L. Ying.Synchrosqueezed wave packet transform for $2 d$ mode decomposition.Submitted on Sep.12, 2012.

[6] F. Auger and P. Flandrin, "Improving the readability of time-frequency and time- scale representations by the reassignment method," IEEE Transactions on Signal Processing, vol. 43, no. 5, pp. 1068-1089, 1995.

[7] E. Cand es, L. Demanet, D. Donoho, and L. Ying. Fast discrete curvelet transforms. MultiscaleModel.Simul., 5(3):861 \{899, 2006.\}

[8] E. J. Cand_es and D. L. Donoho. Continuous curvelet transform. II. Discretization andframes. Appl. Comput. Harmon. Anal., 19(2):198\{222, 2005\}.

[9] Guo, X. Chen, S. Wang, X. Li and R. Liu, "Fault diagnosis of wind turbine using local mean decomposition and synchrosqueezing transforms," 2016 IEEE International Instrumentation and Measurement Technology Conference Proceedings, Taipei,pp.1-6, 2016, doi: 10.1109/I2MTC.2016.7520584

[10] R.Kumar,P.Sumathi and A.Kumar, "Synchrosqueezing Transform-Based Frequency Shifting Detection for Earthquake-Damaged Structures," in IEEE Geoscience and Remote Sensing Letters, vol.14,no.8,pp.1393-1397,Aug.2017.doi: 10.1109/LGRS.2017.2714428

[11] Pallavi R. Patil, A. H. Karode and Dr. S. R. Suralkar, Human Skin Detection Using Image Fusion. International Journal of Electronics and Communication Engineering and Technology, 8(4), pp. 13-21. 2017

[12] Rachana Dhannawat, Tanuja Sarode and H. B. Kekre, Kekre's Hybrid Wavelet Transform Technique with Dct, Walsh, Hartley and Kekre's Transform for Image Fusion, International Journal of Computer Engineering and Technology (IJCET), Volume 4, Issue 1, pp. 195-202, January-February (2013)

[13] Kunal Kishore Pandey and Shekhar R Suralkar, Medical Image Fusion Using Curvelet Transform, International Journal of Electronics and Communication Engineering \& Technology (IJECET), Volume 4, Issue 3, pp. 193-201, May - June, 2013

[14] Jaspreet Kaur and Chirag Sharma, Multimodality Medical Image Fusion Using Improved Contourlet Transformation with Log Gabor Filters, International Journal of Electronics and Communication Engineering \& Technology (IJECET), Volume 4, Issue 2, pp. 383-389, MarchApril, 2013

[15] Asst Prof. Pravin J Barad, Multiresolution Image Fusion Using Nonsubsampled Contourlet Transform, International Journal of Electronics and Communication Engineering \& Technology (IJECET), Volume 5, Issue 7, pp. 15-24, July (2014)

[16] Benayad NSIRI, Salma NAGID1 and Najlae IDRISSI, New Approach to Multispectral Image Fusion Based on a Weighted Merge, International Journal of Electronics and Communication Engineering \& Technology (IJECET), Volume 4, Issue 1, pp. 25-34, January- February (2013)

[17] Dr. Sudeep D. Thepade and Mrs. Jyoti S. Kulkarni, Novel Image Fusion Techniques using Global and Local Kekre Wavelet Transforms, International Journal of Computer Engineering and Technology (IJCET), Volume 4, Issue 1, pp. 89-96, January- February (2013). 